\title{
Use of Platelet-Rich Fibrin for the treatment of gingival recessions: A systematic review and meta-analysis
}

Richard J Miron ${ }^{1 *}$, Vittorio Moraschini2*, Massimo Del Fabbro ${ }^{3,4}$, Adriano Piattelli5, Michael A Pikos', Masako Fujioka-Kobayashi ${ }^{7}$, Yufeng Zhang ${ }^{8}$, Nikola Saulacic ${ }^{9}$, Benoit Schaller ${ }^{7}$, Tomoyuki Kawase ${ }^{9}$, Raluca Cosgarea ${ }^{10,11}$, Soren Jepsen ${ }^{11}$, Delia Tuttle $^{12}$, Mark Bishara ${ }^{13}$, Luigi Canullo ${ }^{14}$, Meizi Eliezer ${ }^{1}$, Andreas Stavopoulos ${ }^{15}$, Yoshinori Shirakata ${ }^{16}$, Alexandra Stahli ${ }^{1}$, Reinhard Gruber ${ }^{17}$, Ondine Lucaciu ${ }^{18}$, Sofia Aroca $^{1}$, Hom-Lay Wang ${ }^{19}$, Anton Sculean ${ }^{1}$

${ }^{1}$ Department of Periodontology, University of Bern, Bern, Switzerland

${ }^{2}$ Department of Oral Surgery, Dentistry School, Fluminense Federal University, Niterói, Rio de Janeiro, Brazil

${ }^{3}$ Department of Biomedical, Surgical, and Dental Sciences, University of Milan, Milan, Italy

${ }^{4}$ IRCCS Orthopedic Institute Galeazzi, Milan, Italy

${ }^{5}$ Department of Applied Sciences of Oral and Dental Disease, G. d'Annunzio University of Chieti-Pescara (Ud'A)(Italy)

${ }^{6}$ Private Practice, Pikos Institute, Trinity, Florida, USA

${ }^{7}$ Department of Cranio-Maxillofacial Surgery, University of Bern, Bern, Switzerland

${ }^{8}$ Department of Oral Implantology, University of Wuhan, China

${ }^{9}$ Division of Oral Bioengineering, Institute of Medicine and Dentistry, Niigata University, Niigata, Japan

10 University luliu Hatieganu, Department of Prosthetic Dentistry, Cluj-Napoca, Romania.

${ }^{11}$ Department of Periodontology, Operative and Preventive Dentistry,

University of Bonn, Bonn, Germany

${ }^{12}$ Division Private practice, Canyon Lake Dental Office, Lake Elsinore, California, USA

${ }^{13}$ Division Private practice, West Bowmanville Family Dental, Bowmanville, Ontario, Canada

14 Independent researcher, Rome, Italy.

15 Department of Periodontology, University of Geneva, Switzerland

16 Department of Periodontology, Kagoshima University Graduate School of Medical and Dental Sciences, Kagoshima, Japan.

17 Department of Oral Biology, University of Vienna, Vienna, Austria

18 Department of Oral Rehabilitation, University of Medicine and Pharmacy "Iuliu Hatieganu", Cluj-Napoca, Romania.

${ }^{19}$ Department of Periodontics and Oral Medicine, University of Michigan, Ann Arbor, Michigan, USA.

${ }^{*}$ Authors contributed equally 


\section{Corresponding author}

Richard J Miron

Department of Periodontology

University of Bern

Bern, Switzerland

richard.miron@zmk.unibe.ch

Running Title: Use of PRF for the treatment of gingival recessions

Summary: The use of platelet-rich fibrin (PRF) membranes has been shown to additionally improve the clinical outcomes of root coverage in Miller Class I and II gingival recessions compared to the use of coronally advanced flap (CAF) alone. No change in keratinized mucosa width (KMW) or reduction in pocket depths was reported. Compared to the $\mathrm{CAF}+$ connective tissue graft (CTG) group, a statistically significant increase in root coverage (RC) and KWM was observed in the CAF+CTG group. No differences between PRF and EMD or between PRF and AM were noted for all investigated parameters. In conclusion, the data indicate that PRF improves RC and clinical attachment level (CAL) compared to CAF alone; however, PRF did not improve KWT. It may therefore be recommended that in cases with deficiencies in baseline KWT, CAF+CTG may preferentially lead to better clinical outcomes. 


\begin{abstract}
Background: Platelet-rich fibrin (PRF) has been utilized in regenerative dentistry for over 15 years, mainly favoring the healing of soft tissues. Interestingly, over 10 years have now passed since the first randomized clinical studies reported on its use in gingival recessions. The aim of this systematic review and meta-analysis was to compare the use of PRF with that of other commonly utilized treatment modalities for root coverage procedures.
\end{abstract}

Methods: Articles published until September 2019 were electronically searched in four databases without language restrictions. The eligibility criteria comprised randomized controlled trials (RCTs) with a follow-up period of 6 months or more that compared the performance of PRF with that of other modalities in the treatment of Miller Class I or II gingival recessions. Studies were classified into 5 categories as follows: 1) coronally advanced flap (CAF) alone vs CAF+PRF, 2) $\mathrm{CAF}+$ connective tissue graft (CTG) vs $\mathrm{CAF}+\mathrm{PRF}, 3) \mathrm{CAF}+$ enamel matrix derivative (EMD) vs $\mathrm{CAF}+\mathrm{PRF}, 4)$ $\mathrm{CAF}+\mathrm{CTG}$ vs $\mathrm{CAF}+\mathrm{CTG}+\mathrm{PRF}$ and 5) $\mathrm{CAF}+$ amnion membrane (AM) vs $\mathrm{CAF}+\mathrm{PRF}$. Studies were evaluated for root coverage (RC) (primary outcome), clinical attachment level (CAL), keratinized mucosa width (KMW) and pocket depth (secondary outcomes).

Results: From 976 articles identified, 17 randomized clinical trials were included. The use of PRF significantly increased RC and CAL compared to the use of CAF alone. No significant change in KMW or reduction in pocket depths was reported. Compared to PRF, CTG led to statistically significant increase in KTW and RC. The use of PRF in combination with CTG led to statistically significant improvements in RC and CAL, while no added benefits were reported in KTW. No statistically significant differences were reported between the $\mathrm{CAF}+\mathrm{PRF}$ and $\mathrm{CAF}+\mathrm{EMD}$ groups or between the $\mathrm{CAF}+\mathrm{PRF}$ and $\mathrm{CAF}+\mathrm{AM}$ groups for any of the investigated parameters. Nevertheless, only one RCT was found for each of the latter three comparisons.

Conclusions: The present systematic review and meta-analysis suggested that the use of PRF for root coverage procedures improved RC and CAL compared to the use of $\mathrm{CAF}$ alone. While similar outcomes were observed between $\mathrm{CAF}+\mathrm{PRF}$ and $\mathrm{CAF}+\mathrm{CTG}$ for CAL and PD change, the latter group led to statistically better outcomes in RC and KTW. In summary, PRF may represent a valid low-cost and easy-to-obtain regenerative modality for the treatment of gingival recessions with adequate baseline KTW.

Key Words: Gingival recession; periodontal plastic surgery; platelet-rich fibrin; evidence-based dentistry
Commentato [m1]: Either KTW or KMW throughout.I also found sone KWT, please fix

Commentato [m2]: I would remove all of this as it is not
a conclusion but results
Commentato [m3]: user-friendly
Commentato [m4]: Miller's class I-II gingival recession.




\section{Introduction}

Periodontal surgery for the management of gingival recessions plays a continuously growing role in modern dentistry due to the increasing demand for optimal esthetics. Studies conducted in the United States have now demonstrated that approximately $90 \%$ of the population reports having at least one tooth with a 1-mm recession by age 60 , with up to $40 \%$ displaying recessions greater than $3 \mathrm{~mm} .^{1,2}$ Furthermore, untreated recessions may be associated with difficulties in performing adequate oral hygiene measures and subsequent risk increase for further attachment loss, root caries and dentin hypersensitivity. ${ }^{3}$

Ideally, such complications necessitate regenerative procedures aimed at regenerating lost tissues. The ultimate goal of root coverage procedures is a resolution of the defect by providing complete root coverage, with ideal keratinized and attached tissue with a seamless esthetic transition with neighboring tissues. ${ }^{4}$

Multiple periodontal plastic surgical procedures with a variety of biomaterials have been proposed to correct these mucogingival deformities and thus rebuild the lost attachment apparatus. Secondarily, increases in attached and keratinized tissue following such regenerative procedures have been shown to favor the long-term maintenance of periodontal tissues. Traditionally, connective tissue grafts (CTGs) from the palate in combination with coronally or laterally positioned flaps have been utilized. Nevertheless, alternative treatment options, including various biomaterials and/or bioactive agents, have been proposed over the years with the aim of lowering patient morbidity. ${ }^{4,5}$

A variety of collagen-based membranes and dermal tissue derivatives from either allograft or xenograft origin have been brought to market for the management of gingival recessions. While these substitute materials provide an excellent three- 
dimensional matrix for the migration and proliferation of fibroblasts, reported disadvantages have included limited regenerative potential as well as a lack of longterm keratinization of tissues within the grafted regions. ${ }^{6}$ In an attempt to increase the potential of membranes, a variety of commercial membranes derived from placental tissues (amnion) have recently been brought to market, although long-term clinical data remain scarce.

Similarly, another strategy has been the use of regenerative growth factors, either utilized alone or in combination with collagen membranes or CTGs, to stimulate the regenerative potential of fibroblasts within the defect area. One commonly utilized bioactive agent for the treatment of gingival recessions has been the use of enamel matrix derivative (EMD) derived from porcine origin. Results from animal and human studies have shown that EMD leads to positive clinical and histological outcomes when combined with a coronally advanced flap (CAF) procedure. ${ }^{7,8}$ Similarly, the use of recombinant human growth factors such as rhPDGF (Gem21) has also been successfully utilized in the treatment of gingival recessions.(need refs e.g. Mc Guire MK et al. JOP 2009, 2014)

Over the past decade, blood concentrates have been proposed as a means to further speed tissue regeneration in dentistry and medicine. Originally, platelet-rich plasma (PRP) was utilized as the first generation blood concentrate with widespread use, particularly in the field of maxillofacial surgery..$^{9}$ Over time, one of the reported drawbacks included its use of anticoagulants, or known inhibitors of clot formation, thereby decreasing the long-term release of growth factors and ultimately diminishing its regenerative potential. ${ }^{10}$ For these reasons, the use of PRP has gradually decreased over the years, and its application in root coverage and muco-gingival procedures has never been well adapted during routine periodontal surgical procedures. 
Platelet-rich fibrin (PRF) has since been developed as a second-generation platelet concentrate with the aim of anticoagulant removal. ${ }^{11-15}$ Following centrifugation, a fibrin-dense membrane is produced with entrapment of host platelets and leukocytes shown to favor the slow and gradual release of growth factors. A number of systematic reviews have thoroughly documented the use of PRF in regenerative dentistry, where it has been shown to particularly favor soft tissue healing over hard tissue healing. ${ }^{13,16,17}$ Furthermore, the use of PRF has also been evaluated for root coverage procedures in randomized clinical studies. ${ }^{18-20}$ Therefore, the aim of this systematic review and meta-analysis was to evaluate the current evidence regarding the use of PRF for the treatment of Miller Class I or II gingival recessions in comparison to other treatment options. Studies were gathered into 5 categories as follows: 1) CAF alone vs $\mathrm{CAF}+\mathrm{PRF}$, 2) $\mathrm{CAF}+\mathrm{CTG}$ vs $\mathrm{CAF}+\mathrm{PRF}$, 3) $\mathrm{CAF}+\mathrm{PRF}$ vs $\mathrm{CAF}+\mathrm{EMD}$, 4) $\mathrm{CAF}+\mathrm{CTG}$ vs $\mathrm{CAF}+\mathrm{CTG}+\mathrm{PRF}$ and 5) $\mathrm{CAF}+\mathrm{AM}$ vs $\mathrm{CAF}+\mathrm{PRF}$. The primary outcome evaluated was root coverage (RC). Secondary outcomes included clinical attachment level (CAL), keratinized mucosa width (KMW) gain and pocket depths. 


\section{Materials and Methods}

\section{Protocol and registration}

This systematic review (SR) was undertaken by following the recommendations of the

Cochrane Handbook for Systematic Reviews of Interventions ${ }^{21}$, and the PRISMA ${ }^{22}$ checklist, in order to increasing the quality and transparency of the study reporting. The review was recorded in the PROSPERO database under number CRD42019139709.

Focused question (based on PICO criteria) ${ }^{23}$

What are the clinical outcomes $(O)$ of using PRF $(I)$ to improve the treatment of Miller

Class I or II gingival recessions $(P)$ compared to other treatment options $(C)$ ?

\section{Outcome measures}

The primary outcome variable was the change in the percentage of root coverage (RC). The secondary outcome variables were clinical attachment level (CAL), keratinized mucosa width (KMW), and probing depth (PD).

\section{Search strategy}

Electronic searches were conducted in PubMed/MEDLINE, Cochrane Central Register of Controlled Trials, Web of Science, and Embase for articles that were published until September 2019 without restrictions on dates or language. In addition, manual searches of the following journals were performed: Journal of Periodontology, Journal of Clinical Periodontology, Journal of Periodontal Research, and International Journal of Periodontics \& Restorative Dentistry. A search of the grey literature through the Literature Report ${ }^{24}$ and OpenGrey databases ${ }^{25}$ was also conducted. Finally, we evaluated the reference lists (cross-referencing) of previous reviews and all potentially 
included studies using MeSH terms, keywords, and other free terms related to the following: "gingival recession", "gingival recessions", "keratinized gingiva”, "Miller

Class I", "Miller Class II", "plastic surgery", "periodontal plastic surgery" “mucogingival surgery”, "muco-gingival surgery", "root coverage”, "platelet-rich fibrin", and "PRF".

\section{Eligibility criteria and study selection process}

This SR included RCTs and prospective controlled trials with follow-up periods of 6 months or more that compared the performances of PRF with CAF alone or in combination with other biomaterials in patients with Miller Class I or II gingival recessions. ${ }^{26}$ The exclusion criteria included animal studies, retrospective cohort studies, in vitro studies, case series, case reports, and reviews. In addition, studies of volunteers with decompensated metabolic disorders or active periodontal disease were also excluded. All studies had to include the use of PRF produced in a centrifuge in standard silica-coated plastic tubes and/or glass tubes.

The process of searching for and selecting the studies was conducted in duplicate by two authors (V.M. and C.F.M.). First, the titles and abstracts were carefully evaluated, followed by the thorough assessment of the potential articles according to the eligibility criteria of this SR. Possible disagreements were resolved when the two authors reached a consensus.

\section{Data synthesis}

The following data, when available, were extracted from the included studies: authors, study design, follow-up, number of treated recessions, number of subjects, age range, gender, number of smokers, Miller class, site of recessions, surgical technique, mean 
difference (MD) in RC between baseline and final follow-up, CAL, KMW, PD, centrifugation system, volume of blood drawn, and centrifugation parameters. Each of the included randomized clinical trials was then grouped into one of the following 5 categories: 1) $\mathrm{CAF}$ alone vs $\mathrm{CAF}+\mathrm{PRF}$, 2) $\mathrm{CAF}+\mathrm{CTG}$ vs $\mathrm{CAF}+\mathrm{PRF}, 3) \mathrm{CAF}+\mathrm{PRF}$ vs $\mathrm{CAF}+\mathrm{EMD}$, 4) $\mathrm{CAF}+\mathrm{CTG}$ vs $\mathrm{CAF}+\mathrm{CTG}+\mathrm{PRF}$ and 5) $\mathrm{CAF}+\mathrm{AM}$ vs $\mathrm{CAF}+\mathrm{PRF}$.

\section{Assessments of the risk of bias}

The risk of bias analysis was performed by two authors (V.M. and C.F.M.). The Cochrane Collaboration's tool for assessing risk of bias ${ }^{27}$ was used for RCTs. Each study was analyzed in relation to six criteria: sequence generation, allocation concealment, blinding, incomplete outcome data, selective outcome reporting, and other sources of bias. Studies were classified as having a low, medium, or high risk of bias when they met all, all but one, or all but two or more criteria, respectively.

\section{Statistical analysis}

The continuous variables (RC, CAL, KMW, and PD) of the included studies were categorized into groups and subgroups and analyzed in a meta-analysis using Review Manager software (version 5.2.8, Copenhagen, Denmark, 2014).

The estimates of the intervention effects (MD) were expressed as percentages or millimeters with $95 \%$ confidence intervals (CIs). The inverse variance method was used for the random-effects or fixed-effects models, depending on the heterogeneity between the studies. Heterogeneity was assessed using chi-square tests. Values $\leq 25 \%,>25 \% \leq$ $50 \%$, and $\geq 50 \%$ were classified as indicating, respectively, low, moderate, and high heterogeneity ${ }^{8}$. The use of the random-effects model was conducted when heterogeneity was found $(p<0.10)$. In contrast, the fixed-effects model was used in 
cases of low or moderate heterogeneity. The statistical significance level of the effect of meta-analysis was fixed at $P \leq 0.05$.

A funnel plot was drawn for the primary outcome variable to assess publication bias across studies.

\section{Results}

\section{Literature search}

The initial search produced 752 titles from MEDLINE/PubMed, 72 from the Cochrane Central Register of Controlled Trials, 69 from the Web of Science, and 83 from EMBASE. After the first evaluation (title and abstract assessment), 952 articles were excluded. Of the 24 potential articles, 7 studies ${ }^{28-34}$ were excluded after careful reading of the full text, because they did not meet the inclusion criteria. Consequently, 17 studies $^{35-51}$ published between 2009 and 2019 were included in this SR. The reasons for the exclusion of potential studies and the search and selection processes are presented in Figure 1.

\section{Study Characteristics}

The characteristics of the included studies are presented in Table 1. Seventeen RCTs ${ }^{35-}$

51 (eleven $^{35,40-42,44-48,50,51} 15,20-22,24-28,30,31$ with a parallel design and six ${ }^{36-39,43,49}$ with a split-mouth design) were included in this SR. The number of participants in the studies ranged from $10^{41}$ to $40,,^{50}$ with an average sample size of $36 \pm 2.93$ patients. A total of 831 (432 test and 399 control) gingival recessions were treated. All of the studies focused on single or multiple Miller Class I or II recessions. Two studies ${ }^{36,37}$ analyzed only teeth from the anterior maxillary region. The follow-up period ranged from 6 
months ${ }^{35,37-40,42-45,47-51}$ to 12 months $^{36,41,46}$ (mean: 7.05 months). All of the articles

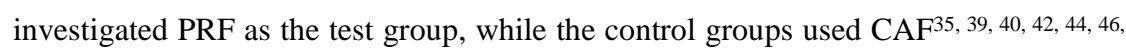
47, 49 CTG $^{37,38,41,43-45,48,50}, \mathrm{EMD}^{36}$, and $\mathrm{AM}^{51}$. Only one study ${ }^{37}$ did not report data on the inclusion of smoking volunteers.

\section{Root coverage}

A random-effects model was used to evaluate the $\mathrm{RC}$ due to the high heterogeneity that was found among the subgroups $\left(P<0.00001 ; I^{2}=86 \%\right)$. With respect to the overall effect, the use of PRF did not differ significantly $(P=0.11)$ from the use of CAF, CTG, EMD, and AM, with an MD of 4.28 (95\% CI: -0.93 to 9.49). In the subgroup analysis, it was observed that PRF significantly improved RC compared to CAF alone $(P=0.05)$ and when associated with CTG $(P<0.00001)$, with an MD of 9.60 (95\% CI: 0.00 to 19.1) and 9.70 (95\% CI: 6.09 to 13.3), respectively. However, when PRF was compared to CTG, there was a statistically significantly greater RC favoring CTG, with an MD of -3.96 (95\% CI: -7.84 to -0.08 ) (Figure 2). The funnel plot demonstrated an asymmetric distribution, indicating a high risk of publication bias (Figure 3). Most of the asymmetry can be attributed to studies involving the CAF vs. PRF group. The sensitivity analysis (exclusion of outliners) suggests that the divergence between the size of the sample groups may favor an increased possibility of publication bias.

\section{Clinical attachment level}

For CAL, the random-effects model was used due to the moderate heterogeneity among the analyzed subgroups $\left(P=0.01 ; I^{2}=47 \%\right)$. The use of PRF alone improved CAL gain compared to the use of CAF. No differences were found when comparing PRF vs.

CTG, PRF vs. EMD or PRF vs. AM. When PRF was combined with CTG, a 
significantly greater CAL was observed compared to CTG alone, with an MD of -0.28 (95\% CI: -0.47 to -0.09 ) (Figure 4).

\section{Keratinized mucosa width}

One study ${ }^{47}$ did not report data on KMW. The random-effects model was utilized for the evaluation of an increase in KMW due to the high heterogeneity among the analyzed subgroups $\left(P<0.0001 ; I^{2}=66 \%\right)$. Regarding the overall effect, the use of PRF did not significantly $(P=0.30)$ increase the KMW when compared to the use of CAF. Only when CTG was compared to PRF $(P=0.03)$ there was a statistically significant improvement favoring the CTG group, with an MD of -0.50 (95\% CI: -0.95 to -0.05 ) (Figure 5).

\section{Probing depth}

Two studies ${ }^{45,47}$ did not evaluate PD. The random-effects model was used to evaluate PD variation due to the moderate heterogeneity observed $\left(P=0.07 ; I^{2}=37 \%\right)$. The use of PRF did not significantly alter PD compared to the use of other biomaterials $(P=$ 0.43 ), with an MD of -0.03 (95\% CI: -0.11 to 0.05 ) (Figure 6).

\section{Assessments of the risk of bias}

The results of the risk of bias analyses of the included studies are presented in Table 2 . None of the studies obtained the highest score in the analysis. Three studies ${ }^{42,48,51}$ were classified as low risk, while nine $35,36,38-41,43,46,50$ and five ${ }^{37,44,45,47,49}$ studies were classified as moderate and high risk, respectively.

\section{Discussion}


To the best of the authors' knowledge, this is the first systematic review and metaanalysis investigating the use of PRF in randomized clinical studies compared to all treatment modalities published to date on the topic. The findings from the present SR were collected to more specifically address the clinical outcomes and recommendations for PRF with respect to its use in the treatment of Miller Class I and II gingival recessions compared to the use of other standard modalities currently utilized in the field. Overall, the majority of studies to date compared the use of PRF with CAF vs $\mathrm{CAF}$ alone or $\mathrm{CAF}+\mathrm{CTG}$ vs $\mathrm{CAF}+\mathrm{PRF}$ (Table 1). Furthermore, additional studies were gathered comparing $\mathrm{CAF}+\mathrm{EMD}$ vs $\mathrm{CAF}+\mathrm{PRF}, \mathrm{CAF}+\mathrm{CTG}$ vs $\mathrm{CAF}+\mathrm{CTG}+\mathrm{PRF}$, and $\mathrm{CAF}+\mathrm{AM}$ vs $\mathrm{CAF}+\mathrm{PRF}$. Below, we highlight and discuss the summary of evidence from the current categories and further discuss the strengths and limitations of each comparative analysis (Table 3).

\section{CAF alone vs CAF+PRF}

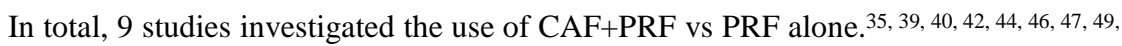

51 The meta-analysis demonstrated that the addition of PRF to CAF significantly improved both root coverage and CAL gains. No advantage was found between the groups for changes in either PD or KWT. Interestingly, one study demonstrated a statistically significant improvement in RC in the control group, in which CAF alone was used, compared to the CAF+PRF group. ${ }^{52}$ All other studies demonstrated improvement in root coverage in the $\mathrm{CAF}+\mathrm{PRF}$ group (Table 1). A study conducted by Padma et al. demonstrated $100 \%$ root coverage following a 6-month healing period. ${ }^{39}$ Furthermore, significant improvement in CAL was also reported in that study. ${ }^{39}$ Interestingly, a number of studies have demonstrated an approximately 10-25\% increase in RC, although this increase is not statistically significant (Table 1). This lack 
of significance was most likely due to the low number of included patients, thus validating the need for meta-analysis. In general, the meta-analysis demonstrated an approximately $10-15 \%$ higher improvement in terms of root coverage when PRF was utilized in combination with CAF compared to the use of CAF alone. Notably, the addition of PRF did not improve KWT. Therefore, it is clinically recommended that the use of PRF does not sufficiently increase KWT as a biomaterial/regenerative agent.

Reported differences in final outcomes among studies may be due to the investigated parameters. Currently, there are no guidelines with respect to using PRF for treatment of Miller Class I and II gingival recessions in terms of the ideal thickness of PRF, the number of PRF membranes needed per site, and/or the ideal surgical technique with respect to its use. Interestingly, one study utilized only $5 \mathrm{~mL}$ of total blood volume collected yet maintained the use of the standard 2700 RPM for 12-minute protocols. ${ }^{47}$ Notably, changes in total blood volume utilized during the centrifugation process changes not only the final cell concentration of PRF membranes but also the centrifugation g-forces produced at the actual PRF clot. The final platelet/leukocyte concentration that may have resulted following such modifications remains poorly investigated, which may explain the lack of improvement in RC in certain studies. The proper use of centrifugation protocols and their respective centrifugation tubes is therefore of utmost importance to avoid potential bias in the results.

\section{CAF+CTG vs CAF+PRF}

In total, 7 studies investigated the use of CAF+CTG vs CAF+PRF. 37, 38, 41, 43-45, 48 . Findings from the meta-analysis revealed that a statistically significant advantage was observed in the CAF+CTG group for RC compared to the CAF+PRF group (Table 2). Furthermore, a number of studies concluded that the use of CTG led to statistically 
significant increase in keratinized tissue thickness compared to the use of PRF. Thus, it may be concluded that, compared to the use of PRF, the use of CTG primarily improves the keratinization of tissues, with an increase in both KTW and RC. Jankovic et al. (2012), Oncu et al. (2017), and Culhaoglu et al. (2018) all demonstrated a statistically significant advantage in KTW in the CAF+CTG group when compared to the CAF+PRF group. ${ }^{43}, 48$ In the later study, only a marginal increase in KTW was observed in the CAF+PRF group $(0.19 \mathrm{~mm})$, whereas the use of CAF+CTG led to a 2.24-mm increase in KTW compared to the findings in controls (over a 10-fold increase). Thus, the use of CTG is seems to be better suited to treat gingival recessions associated with deficiencies in either KTW or keratinized tissue thickness.

\section{$\mathrm{CAF}+\mathrm{CTG}$ vs CAF+CTG+PRF}

Despite the widespread use of PRF, only one study has investigated its use in combination with CTG. ${ }^{50}$ In that study, a statistically significant increase in RC and CAL was observed, although no change in PD or KTW was observed. Future clinical studies are therefore needed to further investigate whether the addition of PRF to CTG provides additional clinical benefit.

\section{CAF+EMD vs CAF+PRF}

Only one randomized clinical study has evaluated the use of CAF in combination with EMD when compared to PRF. ${ }^{36}$ Following a 12-month healing period, no difference was observed in any of the investigated parameters. ${ }^{36}$

\section{$\mathrm{CAF}+\mathrm{AM}$ vs CAF+PRF}

One study compared the use of CAF+AM vs that of CAF+PRF. ${ }^{51}$ Once again, although 
a $20 \%$ increase in root coverage was observed in the CAF+PRF group, the large standard deviation combined with the limited number of clinical studies demonstrated no significant differences in any of the investigated parameters. Future research is needed.

\section{Use of PRF in pain management}

One interesting finding reported in several studies was the highlighted decrease in patient morbidity/pain scores associated with the use of PRF. In total, 5 studies have reported advantages in the use of PRF for lowering pain scores compared to the use of

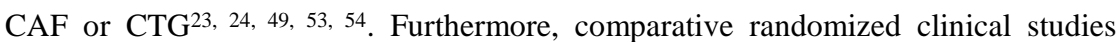
utilizing VAS scores have further demonstrated that the use of PRF placed within the donor site of CTG leads to a statistically significant reduction in postoperative pain. ${ }^{55-}$ 57 Therefore, the use of PRF provides an improvement in patient-reported morbidity at the donor site compared to the use of CAF alone, as well as during the harvesting of CTG at the recipient site.

\section{Implications for Clinical Practice and Future Direction}

A number of important findings were observed within the present systematic review and meta-analysis. In general, the use of PRF in combination with CAF procedures lead to statistically significant improvements in RC and CAL gains compared to the use of CAF alone. Nevertheless, it is important to highlight the fact that relatively no change in KTW or keratinized tissue thickness was observed when PRF was utilized, which therefore highlights the fact that PRF alone is not sufficient to improve areas with deficiencies in keratinized tissues.

Conventionally, CTG has been the gold standard in the coverage of gingival 
recessions. Although several randomized clinical studies highlighted within the present systematic review demonstrated comparable results in RC and CAL, it is important to note that the use of CTG statistically significantly and markedly improved KTW. The meta-analysis therefore confirms the need to utilize CTG for root coverage procedures demonstrating minimal KTW values at baseline. To date, no study has provided guidelines and/or recommendations identifying the minimum KTW required from grafting with PRF alone vs with CTG. This missing data would greatly benefit patient care.

Several drawbacks were observed within the present systematic review. First, there exists no long-term data with even a 2-year follow-up for sites grafted with PRF. Although randomized clinical studies on the topic have now been performed for over a decade, no single study has evaluated the long-term follow-up of patients beyond 18 months. This important missing data remains a priority for future research.

Second, very rarely has surgical technique been discussed as a potential means of altering clinical outcomes. As such, variability in the results obtained has been observed, yet little discussion is generally provided by the authors regarding the surgical protocols/guidelines that may further affect surgical outcomes when grafting with PRF. Questions as simple as "How many PRF membranes per tooth should be utilized for recession coverage?" remain vaguely answered, and substantial further research on the topic needed. Similarly, surgical technique has been more recently discussed with respect to whether PRF should be utilized with CAF, tunneling or vestibular incisions. While each clinical study reports on surgical procedures, studies performed over the upcoming decade should focus more specifically on necessary technical guidelines while grafting with PRF.

Another highly relevant topic of focus in recent years has been the impact of 
the centrifugation device and protocol used. Generally speaking, the production of PRF can be achieved with an array of centrifugation devices, although several recent studies have demonstrated various advantages/disadvantages with different systems. Recently, Takahashi et al. showed that a fixed-angle centrifuge leads to cell accumulation entirely on the back walls of PRF tubes (distal surface), and membranes are created with an uneven distribution of cells throughout the PRF clot. ${ }^{58}$ Furthermore, it was also recently demonstrated that compared to horizontal centrifugation, fixed-angle centrifugation of PRF led to a 4-fold reduction in the ability to concentrate leukocytes within PRF. ${ }^{59}$ While little comparative work exists on the topic to date, studies over the upcoming years will ideally further elucidate the role of centrifugation protocols and devices in the final clinical outcomes.

Another interesting topic brought to the forefront of basic research endeavors on PRF has also been the very recent discovery that PRF tube quality is highly variable, with many chemical additives, such as silica and/or silicone, being incorporated within the PRF clots following advanced PRF (A-PRF) protocols. ${ }^{60}$ Figure 7 demonstrates the 'leftover' silica particles found in a silica-containing tube following A-PRF protocols. While little information is available regarding the toxicity effects associated with such additives on the final PRF membrane content, formation and tissue integration/inflammation, future preclinical research is necessary to further optimize clinical outcomes and patient satisfaction, especially given the number of clinicians who currently utilize non-FDA- and CE-approved equipment.

Clinical research over the next decade will also benefit from future comparative studies with other currently and commonly utilized biomaterials on the market. For instance, it is surprising that although collagen membranes remain one of the most frequently utilized biomaterials in clinical practice for the treatment of root coverage, 
not a single study has compared its clinical outcomes vs those associated with PRF. An array of studies on this topic would better address this missing information over the coming years. It is also more recently common practice to harvest a liquid-injectable PRF that may be utilized to coat collagen membranes. Future research aimed at investigating whether liquid-PRF improves the biocompatibility and/or regenerative potential of collagen membranes is also needed. Future research comparing PRF with other regenerative agents, such as EMD, AM and rhPDGF, would also be beneficial. Due to the shortage of randomized clinical studies on comparative growth factors, no differences have been reported to date among any of the abovementioned groups. Nevertheless, being entirely autologous, low-cost and easily procurable, PRF proves to be a nice alternative to commonly utilized synthetic or xenogeneic biomaterials.

\section{Conclusions}

The present systematic review and meta-analysis addressed the use of PRF in the treatment of Miller Class I or II gingival recessions. The findings from the metaanalysis revealed that, compared to the use of CAF, the use of PRF significantly increased RC and CAL, although no improvement in KTW was observed. When $\mathrm{CAF}+\mathrm{CTG}$ was compared to $\mathrm{CAF}+\mathrm{PRF}$, no difference in PD and CAL was observed; however, it is important to note that $\mathrm{CAF}+\mathrm{CTG}$ led to a significant improvement in KTW and RC. Therefore, it may be concluded that baseline deficiencies in KTW require the use of CTG for improvements in clinical outcomes. No differences in any of the investigated parameters were reported among the CAF+EMD, CAF+AM, and CAF+PRF groups. PRF also improved patient related outcomes in terms of postoperative discomfort and pain following therapy. 


\section{Table captions}

Table 1. Main characteristics of the included studies.

Table 2. Assessments of the risk of bias of randomized clinical trials.

Table 3. Summary of the comparison of 4 outcomes between the platelet rich fibrin (PRF) group and the other treatment modalities. 


\section{Figure captions}

Figure 1. Flow diagram (PRISMA format) of the screening and selection process.

Figure 2. Forest plot for the event "root coverage"

Figure 3. Funnel plot for the studies reporting the primary outcome "root coverage".

Figure 4. Forest plot for the event "clinical attachment level".

Figure 5. Forest plot for the event "keratinized mucosa width".

Figure 6. Forest plot for the event "probing depth".

Figure 7: In this experiment, PRF clots were produced in 3 different commercially available tubes containing silica. Following centrifugation, the clots were removed, the PRF clots were enzymatically digested, and 'leftover' remaining silica particles were visually assessed with scanning electron microscopy (SEM). SEM observations of silica microparticles were contained in (a) Neotube tubes, (b) Vacuette tubes and (c) Venoject II tubes at low (upper) and high magnification (lower). Note the large incorporation of silica microparticles detached from PRF tube walls into PRF clots. Reprinted with permission from Tsujino et al. 2019. ${ }^{61}$ 


\section{References}

1. Li R, Liu Y, Xu T, et al. The Additional Effect of Autologous Platelet Concentrates to Coronally Advanced Flap in the Treatment of Gingival Recessions: A Systematic Review and Meta-Analysis. BioMed research international 2019;2019.

2. Meshram VS, Lambade PN, Meshram PV, Kadu A, Tiwari MS. The autologous platelet rich fibrin: A novel approach in osseous regeneration after cystic enucleation: A pilot study. Indian Journal of Dental Research 2015;26:560.

3. Serino G, Wennström JL, Lindhe J, Eneroth L. The prevalence and distribution of gingival recession in subjects with a high standard of oral hygiene. Journal of clinical periodontology 1994;21:57-63.

4. Zucchelli G, De Sanctis M. Modified two-stage procedures for the treatment of gingival recession. The European journal of esthetic dentistry : official journal of the European Academy of Esthetic Dentistry 2013;8:24-42.

5. Novaes AB, Jr., Palioto DB. Experimental and clinical studies on plastic periodontal procedures. Periodontology 2000 2019;79:56-80.

6. Jhaveri HM, Chavan MS, Tomar GB, Deshmukh VL, Wani MR, Miller PD, Jr. Acellular dermal matrix seeded with autologous gingival fibroblasts for the treatment of gingival recession: a proof-of-concept study. Journal of periodontology 2010;81:616-625.

7. Shirakata Y, Nakamura T, Shinohara Y, et al. Split-mouth evaluation of connective tissue graft with or without enamel matrix derivative for the treatment of isolated gingival recession defects in dogs. Clinical oral investigations 2019;23:3339-3349.

8. Cairo F, Nieri M, Pagliaro U. Efficacy of periodontal plastic surgery procedures in the treatment of localized facial gingival recessions. A systematic review. Journal of clinical periodontology 2014;41 Suppl 15:S44-62.

9. Agrawal AA. Evolution, current status and advances in application of platelet concentrate in periodontics and implantology. World journal of clinical cases 2017;5:159-171.

10. Del Corso M, Vervelle A, Simonpieri A, et al. Current knowledge and perspectives for the use of platelet-rich plasma (PRP) and platelet-rich fibrin (PRF) in oral and maxillofacial surgery part 1: Periodontal and dentoalveolar surgery. Current pharmaceutical biotechnology 2012;13:1207-1230.

11. Kumar RV, Shubhashini N. Platelet rich fibrin: a new paradigm in periodontal regeneration. Cell and tissue banking 2013;14:453-463.

12. Miron RJ, Fujioka-Kobayashi M, Hernandez M, et al. Injectable platelet rich fibrin (i-PRF): opportunities in regenerative dentistry? Clinical oral investigations 2017.

13. Miron RJ, Zucchelli G, Pikos MA, et al. Use of platelet-rich fibrin in regenerative dentistry: a systematic review. Clinical oral investigations 2017;21:1913-1927.

14. Lourenco ES, Mourao C, Leite PEC, Granjeiro JM, Calasans-Maia MD, Alves GG. The in vitro release of cytokines and growth factors from fibrin membranes produced through horizontal centrifugation. Journal of biomedical materials research Part A 2018;106:1373-1380.

15. Mourão CFdAB, Calasans-Maia MD, de Mello Machado RC, de Brito Resende RF, Alves GGJO, surgery $\mathrm{m}$. The use of platelet-rich fibrin as a hemostatic material in oral soft tissues. 2018;22:329-333. 
16. Najeeb S, Khurshid Z, Agwan MAS, Ansari SA, Zafar MS, Matinlinna JP. Regenerative Potential of Platelet Rich Fibrin (PRF) for Curing Intrabony Periodontal Defects: A Systematic Review of Clinical Studies. Tissue engineering and regenerative medicine 2017;14:735-742.

17. Miron RJ, Fujioka-Kobayashi M, Bishara M, Zhang Y, Hernandez M, Choukroun J. Platelet-Rich Fibrin and Soft Tissue Wound Healing: A Systematic Review. Tissue engineering Part B, Reviews 2017;23:83-99.

18. Castro AB, Meschi N, Temmerman A, et al. Regenerative potential of leucocyte - and platelet - rich fibrin. Part A: intra - bony defects, furcation defects and periodontal plastic surgery. A systematic review and meta analysis. 2017;44:67-82.

19. Miron RJ, Zucchelli G, Pikos MA, et al. Use of platelet-rich fibrin in regenerative dentistry: a systematic review. 2017;21:1913-1927. SAME AS 13

20. Moraschini V, Barboza Edos S. Use of Platelet-Rich Fibrin Membrane in the Treatment of Gingival Recession: A Systematic Review and Meta-Analysis. Journal of periodontology 2016;87:281-290.

21. Higgins JP, Green S. Cochrane handbook for systematic reviews of interventions: John Wiley \& Sons; 2011.

22. Moher D, Liberati A, Tetzlaff J, Altman DG. Preferred reporting items for systematic reviews and meta-analyses: the PRISMA statement. Annals of internal medicine 2009;151:264-269.

23. Schardt C, Adams MB, Owens T, Keitz S, Fontelo P. Utilization of the PICO framework to improve searching PubMed for clinical questions. BMC medical informatics and decision making 2007;7:16.

24. . Available at: https://www.nyam.org/library/collections-and-resources/greyliterature-report/. Accessed: March 10, 2019.

25. . Available at: http://www.opengrey.eu. Accessed: March 10.

26. Miller Jr P. A classification of marginal tissue recession. Int J Periodont Rest Dent 1985;5:9.

27. Higgins JP, Altman DG, Gøtzsche PC, et al. The Cochrane Collaboration's tool for assessing risk of bias in randomised trials. Bmj 2011;343:d5928.

28. Sambhav J, Rohit R, Ranjana M, Shalabh MJEjohs. Platelet rich fibrin (Prf) and $\beta$-tricalcium phosphate with coronally advanced flap for the management of grade-II furcation defect. 2014;24:269-272.

29. Bajaj P, Pradeep A, Agarwal E, et al. Comparative evaluation of autologous platelet - rich fibrin and platelet - rich plasma in the treatment of mandibular degree II furcation defects: a randomized controlled clinical trial. 2013;48:573581

30. Del Corso M, Sammartino G, Dohan Ehrenfest DM. Re: "Clinical evaluation of a modified coronally advanced flap alone or in combination with a platelet-rich fibrin membrane for the treatment of adjacent multiple gingival recessions: a 6month study". Journal of periodontology 2009;80:1694-1697; author reply 1697-1699.

31. Anilkumar K, Geetha A, Umasudhakar, Ramakrishnan T, Vijayalakshmi R, Pameela E. Platelet-rich-fibrin: A novel root coverage approach. Journal of Indian Society of Periodontology 2009;13:50-54.

32. Singh J, Bharti V. Laterally positioned flap-revised technique along with platelet rich fibrin in the management of Miller class II gingival recession. Dental research journal 2013;10:268-273. 
33. Ramireddy S, Mahendra J, Rajaram V, Ari G, Kanakamedala AK, Krishnakumar D. Treatment of gingival recession by coronally advanced flap in conjunction with platelet-rich fibrin or resin-modified glass-ionomer restoration: A clinical study. Journal of Indian Society of Periodontology 2018;22:45-49.

34. Uzun BC, Ercan E, Tunali M. Effectiveness and predictability of titaniumprepared platelet-rich fibrin for the management of multiple gingival recessions. Clinical oral investigations 2018;22:1345-1354.

35. Aroca S, Keglevich T, Barbieri B, Gera I, Etienne D. Clinical evaluation of a modified coronally advanced flap alone or in combination with a platelet-rich fibrin membrane for the treatment of adjacent multiple gingival recessions: a 6 month study. Journal of periodontology 2009;80:244-252.

36. Jankovic S, Aleksic Z, Milinkovic I, Dimitrijevic B. The coronally advanced flap in combination with platelet-rich fibrin (PRF) and enamel matrix derivative in the treatment of gingival recession: a comparative study. The European journal of esthetic dentistry : official journal of the European Academy of Esthetic Dentistry 2010;5:260-273.

37. Jankovic $\mathrm{S}$, Aleksic Z, Klokkevold P, et al. Use of platelet-rich fibrin membrane following treatment of gingival recession: a randomized clinical trial. The International journal of periodontics \& restorative dentistry 2012;32:e41-50.

38. Eren G, Atilla G. Platelet-rich fibrin in the treatment of localized gingival recessions: a split-mouth randomized clinical trial. Clinical oral investigations 2014;18:1941-1948.

39. Padma R, Shilpa A, Kumar PA, Nagasri M, Kumar C, Sreedhar A. A split mouth randomized controlled study to evaluate the adjunctive effect of platelet-rich fibrin to coronally advanced flap in Miller's class-I and II recession defects. Journal of Indian Society of Periodontology 2013;17:631-636.

40. Thamaraiselvan M, Elavarasu S, Thangakumaran S, Gadagi JS, Arthie T. Comparative clinical evaluation of coronally advanced flap with or without platelet rich fibrin membrane in the treatment of isolated gingival recession. Journal of Indian Society of Periodontology 2015;19:66-71.

41. Tunali M, Ozdemir H, Arabaciota T, Gurbuzer B, Pikdoken L, Firatli E. Clinical evaluation of autologous platelet-rich fibrin in the treatment of multiple adjacent gingival recession defects: a 12-month study. The International journal of periodontics \& restorative dentistry 2015;35:105-114.

42. Bozkurt Dogan S, Ongoz Dede F, Balli U, Atalay EN, Durmuslar MC. Concentrated growth factor in the treatment of adjacent multiple gingival recessions: a split-mouth randomized clinical trial. Journal of clinical periodontology 2015;42:868-875.

43. Oncu E. The Use of Platelet-Rich Fibrin Versus Subepithelial Connective Tissue Graft in Treatment of Multiple Gingival Recessions: A Randomized Clinical Trial. The International journal of periodontics \& restorative dentistry 2017;37:265-271.

44. Kumar A, Bains VK, Jhingran R, Srivastava R, Madan R, Rizvi I. Patientcentered Microsurgical Management of Gingival Recession using Coronally Advanced Flap with Either Platelet-rich Fibrin or Connective Tissue Graft: A Comparative Analysis. Contemporary clinical dentistry 2017;8:293-304.

45. Mufti S, Dadawala SM, Patel P, Shah M, Dave DH. Comparative Evaluation of Platelet-Rich Fibrin with Connective Tissue Grafts in the Treatment of Miller's Class I Gingival Recessions. Contemporary clinical dentistry 2017;8:531-537. 
46. Kuka S, Ipci SD, Cakar G, Yilmaz S. Clinical evaluation of coronally advanced flap with or without platelet-rich fibrin for the treatment of multiple gingival recessions. Clinical oral investigations 2018;22:1551-1558.

47. Dixit N, Lamba AK, Faraz F, Tandon S, Aggarwal K, Ahad A. Root coverage by modified coronally advanced flap with and without platelet-rich fibrin: A clinical study. Indian journal of dental research : official publication of Indian Society for Dental Research 2018;29:600-604.

48. Culhaoglu R, Taner L, Guler B. Evaluation of the effect of dose-dependent platelet-rich fibrin membrane on treatment of gingival recession: a randomized, controlled clinical trial. Journal of applied oral science : revista FOB 2018;26:e20170278.

49. Gupta S, Banthia R, Singh P, Banthia P, Raje S, Aggarwal N. Clinical evaluation and comparison of the efficacy of coronally advanced flap alone and in combination with platelet rich fibrin membrane in the treatment of Miller Class I and II gingival recessions. Contemporary clinical dentistry 2015;6:153160.

50. Keceli HG, Kamak G, Erdemir EO, Evginer MS, Dolgun A. The Adjunctive Effect of Platelet-Rich Fibrin to Connective Tissue Graft in the Treatment of Buccal Recession Defects: Results of a Randomized, Parallel-Group Controlled Trial. Journal of periodontology 2015;86:1221-1230.

51. Agarwal SK, Jhingran R, Bains VK, Srivastava R, Madan R, Rizvi I. Patientcentered evaluation of microsurgical management of gingival recession using coronally advanced flap with platelet-rich fibrin or amnion membrane: A comparative analysis. European journal of dentistry 2016;10:121-133.

52. Aroca S, Keglevich T, Barbieri B, Gera I, Etienne D. Clinical evaluation of a modified coronally advanced flap alone or in combination with a platelet - rich fibrin membrane for the treatment of adjacent multiple gingival recessions: A 6 - month study. Journal of periodontology 2009;80:244-252. SAME AS 35

53. Anilkumar K, Geetha A, Umasudhakar TR, Vijayalakshmi R, Pameela E. Platelet-rich-fibrin: A novel root coverage approach. Journal of Indian Society of Periodontology 2009;13:50.

54. Tunalı M, Özdemir H, Arabacı T, Gürbüzer B, Pikdöken ML, Fıratlı E. Clinical evaluation of autologous platelet-rich fibrin in the treatment of multiple adjacent gingival recession defects: a 12-month study. International Journal of Periodontics \& Restorative Dentistry 2015;35.

55. Femminella B, Iaconi MC, Di Tullio M, et al. Clinical Comparison of PlateletRich Fibrin and a Gelatin Sponge in the Management of Palatal Wounds After Epithelialized Free Gingival Graft Harvest: A Randomized Clinical Trial. Journal of periodontology 2016;87:103-113.

56. Lektemur Alpan A, Torumtay Cin G. PRF improves wound healing and postoperative discomfort after harvesting subepithelial connective tissue graft from palate: a randomized controlled trial. Clinical oral investigations 2019.

57. Ustaoglu G, Ercan E, Tunali M. The role of titanium-prepared platelet-rich fibrin in palatal mucosal wound healing and histoconduction. Acta odontologica Scandinavica 2016;74:558-564.

58. Takahashi A, Tsujino T, Yamaguchi S, et al. Distribution of platelets, transforming growth factor-beta1, platelet-derived growth factor-BB, vascular endothelial growth factor and matrix metalloprotease-9 in advanced platelet- 
rich fibrin and concentrated growth factor matrices. Journal of investigative and clinical dentistry 2019:e12458.

59. Miron RJ, Chai J, Zheng S, Feng M, Sculean A, Zhang Y. A novel method for evaluating and quantifying cell types in platelet rich fibrin and an introduction to horizontal centrifugation. Journal of biomedical materials research Part A 2019.

60. Tsujino T, Masuki H, Nakamura M, et al. Striking Differences in Platelet Distribution between Advanced-Platelet-Rich Fibrin and Concentrated Growth Factors: Effects of Silica-Containing Plastic Tubes. Journal of functional biomaterials 2019;10.

61. Tsujino T, Takahashi A, Yamaguchi S, et al. Evidence for Contamination of Silica Microparticles in Advanced Platelet-Rich Fibrin Matrices Prepared Using Silica-Coated Plastic Tubes. 2019;7:45. 